\title{
10Gb/s Bi-directional Symmetric WDM-PON System based on POLMUX Technique with Polarization Insensitive ONU
}

\author{
Hyun-Do Jung, Nguyen-Cac Tran, Chigo Okonkwo, Eduward Tangdiongga, and Ton Koonen \\ COBRA Research Institute, Eindhoven University of Technology, 5600 MB Eindhoven, The Netherlands \\ E-mail: jhd94@ieee.org
}

\begin{abstract}
We demonstrated $10 \mathrm{~Gb} / \mathrm{s}$ bi-directional symmetric transmission based on polarizationdivision multiplexing with a centralized light source. One of two orthogonal polarization states carries downstream data while the other un-modulated state is used for upstream modulation.

2010 Optical Society of America

OCIS codes: (060.4510) Optical communications; (060.4250 ) Networks
\end{abstract}

\section{Introduction}

Future access networks have to meet the people's demand for high-quality broadband services. Wavelengthdivision multiplexing passive optical networks (WDM-PONs) employing centralized light sources and reflective, wavelength-insensitive modulators at the optical network units (ONUs) are promising candidates to meet these demands due to its ease of upgradability and high capacity. The advantage of this configuration is that wavelength referencing and control are provided by centrally generated continuous-wave $(\mathrm{CW})$ carriers instead of wavelengthspecific or wavelength-tunable sources at the cost-sensitive ONU.

So far, several researches [1-3] have been reported for this configuration. The injection locked FP-LD [1] is attractive because of its wavelength self-management characteristics. However, the transmission performance depends on the wavelength detuning between the injected sliced ASE and a lasing mode of the LD. For a costeffective and colorless transmitter at the ONU, the use of R(eflective)-SOAs provides several advantages. However, its modulation speed is limited at $2.5 \mathrm{~Gb} / \mathrm{s}$ and it needs a special modulation technique [2] to share the downstream optical signal for the uplink. Recently, a reflective transmitter with polarization-division multiplexing (POLMUX) technique [3] has been introduced for the colorless ONU. This configuration uses a polarization beam splitter to separate downstream and upstream optical signal at ONU. However, for cost-effectiveness, it is advantageous to simplify the ONU side. Hence, a polarization dependent device cannot be used.

In this paper, we propose a new architecture for bi-directional symmetric WDM-PON system based on POLMUX with a polarization insensitive and colorless ONU. As shown in Fig.1, one of two optical signals with orthogonal polarization states launched from a central office $(\mathrm{CO})$ carries downstream data while the other unmodulated state (TM) is used for uplink transmission. At ONU, both polarization states are modulated by the upstream data. Consequently, the downlink signal is overlapped with the upstream data in the uplink, but the orthogonal state carries only the upstream data. Due to this orthogonality, it is possible to separate the upstream data by the polarization filtering at the CO. In this configuration, the ONU is completely polarization independent because all polarization dependent devices are located at the CO. In the experiment, we demonstrated bi-directional $10 \mathrm{~Gb} / \mathrm{s}$ symmetric transmission over $25 \mathrm{~km}$ single mode fiber (SMF) link with the proposed technique and achieved error-free transmission at both down- and up-link.

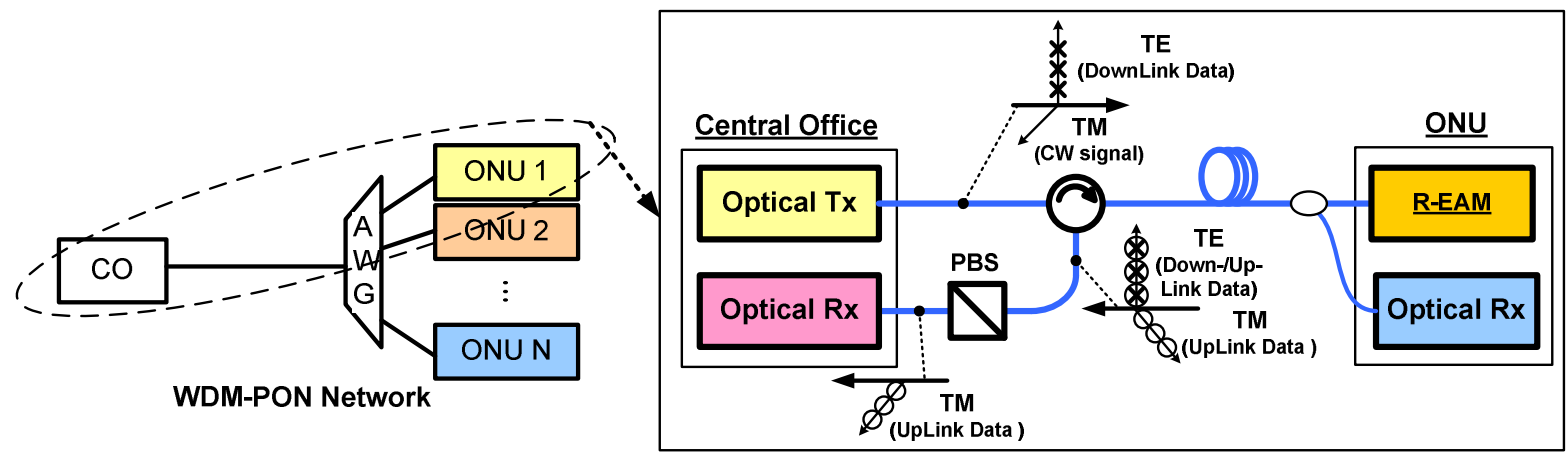

Fig. 1 Proposed WDM-PON system with POLMUX 


\section{Experimental Setup}

In the downstream link, the optical signal is split by the power divider. One portion is modulated by the downstream data $\left(10 \mathrm{~Gb} / \mathrm{s}\right.$, PRBS $\left.2^{31}-1\right)$, and the modulated signal is polarization multiplexed with the un-modulated optical signal by the polarization beam combiner, shown in Fig. 2. Then, the combined optical signals with orthogonal polarization states (TE+TM) are transmitted over $25 \mathrm{~km}$ SMF to the ONU. At ONU, a small portion of signal is tapped for receiving downstream data. The last of the downstream optical signal is injected to R-EAM for the upstream data modulation.

In the ONU, the transmitted optical signal is re-used for the upstream link. The bi-directional optical amplifier before R-EAM compensates for the $25 \mathrm{~km}$ transmission loss, the power splitting loss, and the insertion loss of REAM. The 10Gb/s PRBS electrical signal modulates the transmitted optical signal and generates two orthogonal modulated optical signals, depicted in Fig. 1. To emulate a real situation, a different PRBS pattern, $2^{7}-1$, is used for the upstream modulation. As mentioned in the previous section, the downstream data is overlapped with the upstream data after uplink modulation, but the orthogonal state with $\mathrm{CW}$ optical signal carries only the upstream data. Therefore, after $25 \mathrm{~km}$ upstream transmission, the optical signal with upstream data is easily taken out by the polarization filtering.

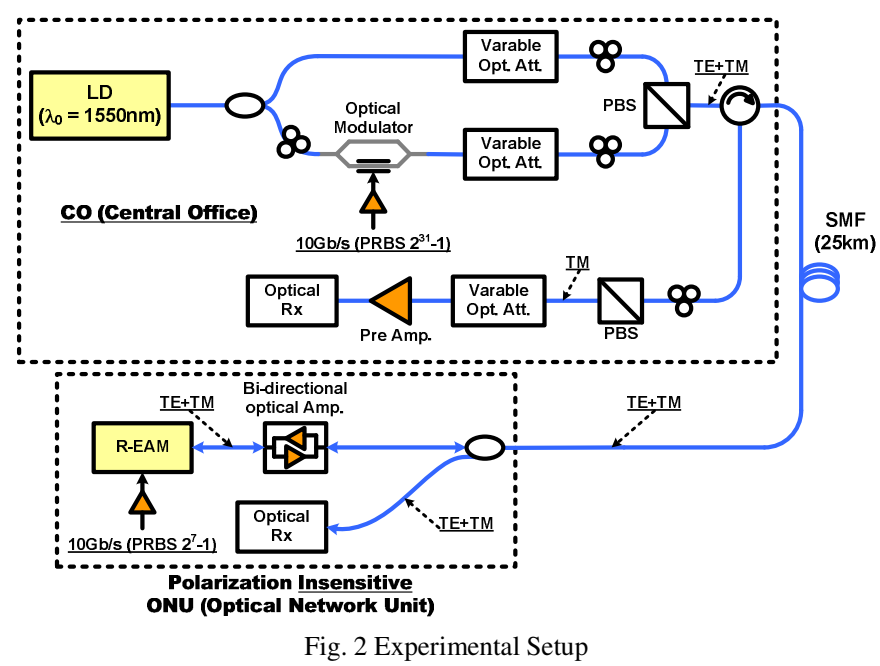

\section{Results}

To evaluate the system performance, we measured the bit-error rate of $10 \mathrm{~Gb} / \mathrm{s}$ PRBS signal at the downstream and upstream link. Figure 3 shows the BER performance of back-to-back and $25 \mathrm{~km}$ transmission. In this demonstration, upstream results are better than the downstream cases. This is because the optical launching condition is optimized at the upstream link where the modulated and un-modulated optical power was $-4 \mathrm{dBm}$ and $2 \mathrm{dBm}$, respectively. If the optical launching powers of two orthogonal signals at CO are the same, it is difficult to achieve error-free transmission at the upstream link because the un-modulated signal cannot get enough modulation depth at R-EAM in ONU. By increasing the CW (un-modulated) optical power, the performance of the uplink improves while that of the downstream degrades due to the DC offset. This trade-off relationship is shown in Fig. 5.

In each case, , the power penalty between $25 \mathrm{~km}$ transmission and back-to-back results are compared. The power penalty is due to the backscattering from the transmission, the crosstalk over bi-directional transmission and the crosstalk between orthogonal channels, which occur during the uplink modulation. Among these factors, the backscattering is the dominant effect. However, the power penalty in the downstream case is higher than that of the upstream case because the polarization filtering reduces the backscattering in the upstream. Fig. 4 shows the eye diagrams measured at the receiver side. In the downstream case shown in Fig. 4(a), the un-modulated CW signal gives the DC offset to the received signal as mentioned above. In the upstream case, the received signals look like noise shown in Fig. 4(b) because both modulated orthogonal signals are detected together. With proper polarization filtering, a clear eye opening in Fig. 4(c) is obtained.

However, in spite of the penalties described above, we achieved error-free transmission in both up- and downstream link with the proposed architecture. 


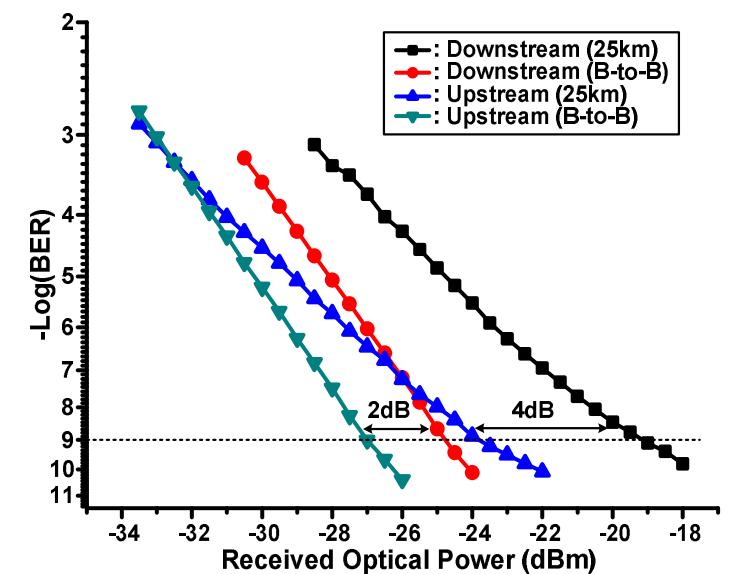

Fig. 3 BER Results (power difference of $2 \mathrm{~dB}$ between TE(downstream) and TM (un-modulated))

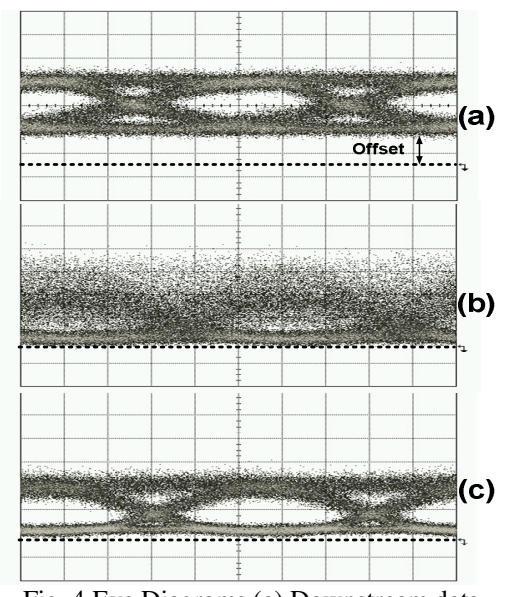

Fig. 4 Eye Diagrams (a) Downstream data,

(b) Up- and Down-stream data (mixed),

(c) Upstream data only,

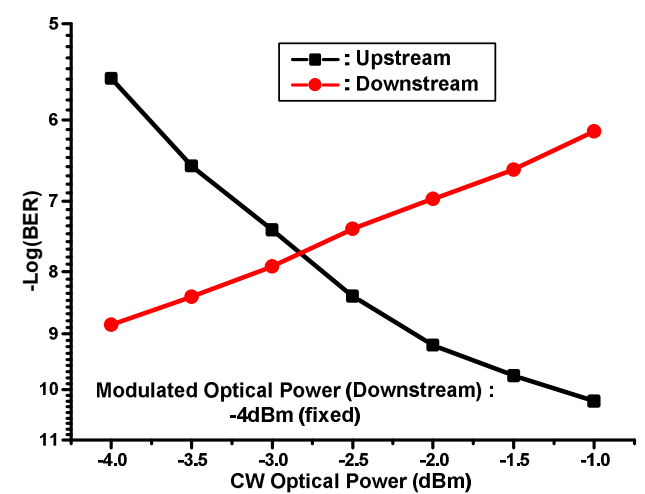

Fig. 5 BER Results (25km) against CW launched optical power (Received Optical Power: -24dBm)

\section{Conclusions}

In this paper, we proposed a new architecture for bi-directional symmetric WDM-PON system based on polarization-division multiplexing technique and successfully demonstrated $10 \mathrm{~Gb} / \mathrm{s}$ bi-directional symmetric transmission with error-free BER performance. In the proposed architecture, the ONU is composed of the polarization insensitive and colourless devices and the polarization filtering to select the upstream signal is placed at the CO so that this system is practical. However, if several ONUs share the same optical wavelength as proposed for WDM/TDM-PON networks, it becomes challenging to realize the network with this idea as fast and complicated polarization tracking is needed. However, in this case, we could consider hybrid WDM/TDM-PON with the proposed idea. By introducing a remote node $(\mathrm{RN})$, which manages several ONUs based on E-/G-PON, between CO and ONUs, we can implement the networks from $\mathrm{CO}$ to $\mathrm{RN}$ based on the proposed architecture and the last of the network is based on E-/G-PON network.

\section{Acknowledgments}

This works has been partially supported by FP7 EU project ALPHA.

\section{References}

[1] H. D. Kim, S.-G. Kang, and C.-H. Lee, “A low-cost WDM source with an ASE injected Fabry-Perot semiconductor laser," IEEE Photon. Technol. Lett., 12, 1067-1069 (2000).

[2] J.-H. Yu, B-W. Kim and N. Kim, "Wavelength Re-use Scheme with Reflective SOA for WDM-PON Link," in proceeding of ICACT 2008, pp. 1704-1710 (2008).

[3] J. Mora, B. Ortega, J. Capmany, and F. Grassi, "Bi-directional Optical Aceess Network based on POLMUX Technique Using Centralized Light Sources," in proceeding of MWP 2009, Th 4.9 (2009). 